\title{
Intentional forgetting of emotional words after trauma: a study with victims of sexual assault
}

\author{
Ines Blix * and Tim Brennen \\ Department of Psychology, Center for the Study of Human Cognition, University of Oslo, Oslo, Norway
}

Edited by:

Mattie Tops, University of Groningen,

Netherlands

Reviewed by:

Miriam Gade, University of Zurich, Switzerland

Martina Rieger, Max Planck Institute

for Human Cognitive and Brain

Sciences/University of Leipzig,

Germany

*Correspondence:

Ines Blix, Department of Psychology, University of Oslo, PO Box 1094,

0317 Oslo, Norway.

e-mail: ines.blix@psykologi.uio.no
Following exposure to a trauma, people tend to experience intrusive thoughts and memories about the event. In order to investigate whether intrusive memories in the aftermath of trauma might be accounted for by an impaired ability to intentionally forget disturbing material, the present study used a modified Directed Forgetting task to examine intentional forgetting and intrusive recall of words in sexual assault victims and controls. By including words related to the trauma in addition to neutral, positive, and threat-related stimuli it was possible to test for trauma-specific effects. No difference between the Trauma and the Control group was found for correct recall of to-be-forgotten (F) words or to-be-remembered (R) words. However, when recalling words from R-list, the Trauma group mistakenly recalled significantly more trauma-specific words from F-list. "Intrusive" recall of F-trauma words when asked to recall R-words was related to symptoms of post-traumatic stress disorder reported on the Impact of Event Scale and the Post-traumatic Diagnostic Scale. The results are discussed in term of a source-monitoring account.

Keywords: intentional forgetting, retrieval, forgetting, rape, directed forgetting, trauma, memory, sexual assault

\section{INTRODUCTION}

After exposure to a trauma people often report intrusive thoughts and memories about the traumatic event (McNally, 2003), and these can be extremely persistent over many years. Intrusive memories or "recurrent recollections" refer to repetitive involuntary memories about the traumatic event. Intrusive memories are experienced as highly disturbing and have the paradoxical property that the more the person tries to suppress or avoid them the more persistent they become (Wegner, 1989). The present study investigated intrusive recall and capacity to intentionally forget in a non-clinical sample of trauma-exposed individuals, and a control group.

A proportion of individuals exposed to trauma develop posttraumatic stress disorder (PTSD), in which intrusive memories and thoughts are core symptoms (DSM-IV). Several models of cognitive functioning in PTSD have been proposed (e.g., Horowitz et al., 1979; Janoff-Bulman, 1992; Ehlers and Clark, 2000; Brewin, 2001; Rubin et al., 2008a). These theories do not only inform us about cognition in clinical PTSD, but can also explain memory processes after trauma-exposure and development of persistent trauma symptoms in the aftermath of trauma. According to Brewin's (2001) and Ehlers and Clark's (2000) models, intrusive memories of the traumatic event arise because these memories are not integrated with the rest of autobiographical memory. Integration in autobiographical memory is prevented by maladaptive strategies like avoidance and suppression of disturbing thoughts.

Rubin et al. (2008a) proposed a memory-based model of PTSD where the development of PTSD symptoms following a trauma depends on the interaction between the event and the memory processes following the event. According to this model, no special memory mechanisms are needed to explain the development of trauma symptoms, or to explain intrusive memories in the aftermath of trauma. Rather what is known about emotion and memory in general can explain the development of trauma symptoms (Berntsen et al., 2008).

Indeed several studies have demonstrated that involuntary memories are not a trauma-specific phenomenon; on the contrary, recurrent involuntary memories are common in everyday life. In the normal population the majority of these are positive (for an overview see Berntsen, 2009). However, in a diary study, students with PTSD symptoms recorded an equal number of positive and negative involuntary memories (Berntsen, 2001). Another diary study with PTSD participants showed that participants with a high level of PTSD symptoms recorded more negative involuntary and voluntary memories than participants with lower levels of PTSD symptoms (Rubin et al., 2008b). Berntsen et al. (2008) argued that emotional stress enhances both encoding of and access to a memory, thus both involuntary and voluntary memory recall will be enhanced for traumatic episodes.

One task that has been used to study encoding and retrieval in the aftermath of trauma is directed forgetting (DF). In a DF task, participants are presented with lists of words, and an accompanying instruction either to remember or to forget the presented words. There are two main versions of the DF task: In the list method DF the Remember or Forget instructions are given after presentation of each list, whereas in the item method the Forget or Remember cue are given after each word. The standard DF effect refers to the fact that one remembers more words that one has been instructed to remember (R-words) compared to words one was instructed to forget (F-words; Johnson, 1994). This DF effect is thought to reflect the ability to voluntarily forget material, and is often referred to as intentional forgetting. Wessel and Merckelbach 
(2006) investigated DF for emotional material in healthy participants. Using a list method DF task, these researchers showed a DF effect of equivalent size for neutral and negative word lists. This study indicates that for healthy participants there is no difference in intentional forgetting of either emotional or neutral material (Wessel and Merckelbach, 2006).

A few studies with trauma-exposed individuals have used the DF task to study recall and forgetting from a perspective hypothesizing that trauma-exposed individuals are characterized by avoidant encoding, that is, a tendency to fail to encode upsetting material. McNally et al. (1998) used an item method DF task to study intentional forgetting of trauma-related words relative to neutral and positive words in sexually abused participant with and without PTSD, and controls. However, in contrast to a hypothesis about avoidant encoding of trauma-related material in PTSD, the results showed no difference between the groups in recall of trauma-related R-words or F-words. On the contrary, but consistent with Rubin et al.'s (2008a) model, the participants remembered trauma words very well, including the words they were instructed to forget. However, the PTSD patients demonstrated lower overall recall rates for positive and negative R-words.

In summary, studies using the DF task to investigate avoidant encoding in trauma-exposed people have not yielded conclusive results, some studies have provided evidence for avoidant encoding (Moulds and Bryant, 2002, 2005), while other studies show that trauma-exposed individuals remember trauma-related material very well (Zoellner et al., 2003; McNally et al., 1998).

Cottencin et al. (2006) investigated DF in trauma-exposed individuals from a perspective arguing that intrusive memories, rather than avoidance and impaired voluntary recall, more correctly characterize cognition in PTSD. They hypothesized that due to a faulty inhibition mechanism, trauma-exposed participants with PTSD would have difficulties inhibiting F-words, compared to nontrauma-exposed controls. In their study, a group of individuals exposed to a variety of traumatic events with a diagnosis of PTSD and a non-trauma-exposed control group completed a version of the item-cued DF task. The DF task comprised immediate conditional recall, where participants were asked to recall R-words, and final unconditional recall where participants were asked to recall both $\mathrm{R}$-words and F-words. The results revealed that in the immediate conditional recall condition the PTSD group recalled fewer R-words compared to the controls. In contrast, the PTSD group recalled more F-words. In the final conditional recall where participants were asked to recall both F- and R-words, a reduced DF effect for the PTSD group was found. The PTSD participants recalled fewer R-words, but there was no difference between groups with respect to recall of F-words.

Intrusive memories are also characteristic for depression (e.g., Brewin et al., 1996), and intrusive memories in depression and PTSD have been found to share the same characteristics (Reynolds and Brewin, 1999). Hence, DF studies with depressed individuals might be informative about intentional forgetting in PTSD. Power et al. (2000) studied DF in clinically depressed, clinically anxious, and healthy controls using a list method DF task, where half of the participants received a Forget cue after the first list and the other half did not. The results showed that the clinically depressed participants in the Forget condition actually recalled more negative words than the clinically depressed participants in the Remember condition. This pattern was not found for positive words. Furthermore this pattern was not found for the clinically anxious participants or the controls. These results suggest a stronger rebound of depression-relevant material in clinical depression. Power et al. (2000) argued that this effect may also explain intrusive memories in PTSD, and suggested a common mechanism in depression and PTSD, involving a stronger rebound effect for aversive personal-relevant information.

In summary, the literature suggests that trauma-exposed individuals, especially those with a diagnosis of PTSD, suffer from intrusive memories about the traumatic event. Moreover, memory after trauma is better characterized by intrusive recollection rather than avoidant encoding and impaired recall of traumarelated memories. As discussed above, intrusive memories in the aftermath of trauma might result from an impaired ability to intentionally forget disturbing material. In line with this Cottencin et al. (2006) found support for deficits in intentional forgetting of neutral material in participants with PTSD. However, in Cottencin et al.'s (2006) study only neutral material was used, and so is silent as to whether different patterns would emerge for emotional and trauma-specific material. Furthermore, Power et al. (2000) reported data showing stronger rebound of depression-relevant material in clinical depression, and argued that the list method DF paradigm would be a good model for studying an enhanced rebound effect and intrusive memories also in PTSD.

In the present study we used a modified version of the list method DF task to study intentional forgetting and intrusive memories in a non-clinical group of trauma-exposed participants and controls. The purpose of the present study was twofold. The first aim was to investigate whether hypothesized deficits in intentional forgetting after trauma are specific to processing of trauma-specific material, or rather reflect a general tendency for all types of material. On the one hand, Cottencin et al.'s (2006) study with trauma-exposed participants with PTSD suggests a general tendency of impaired intentional forgetting in traumaexposed individuals with PTSD. On the other hand, cognitive theories of PTSD (e.g., Brewin et al., 1996; Ehlers and Clark, 2000) suggest that memory deficits in trauma-exposed individuals are specific for processing of material associated with the traumatic episode. Furthermore, these theories predict impaired voluntary recall and enhanced involuntary recall for trauma-specific material in trauma-exposed individuals. Rubin et al.'s (2008a) model is in agreement that involuntary recall should be enhanced, but also predicts enhanced voluntary recall of material associated with the trauma in trauma-exposed individuals.

In order to investigate intrusive recall, a modified version of the list method DF was used. The participants were presented to two lists for words on a computer screen, the first list was followed by an instruction to forget the words, the second was followed by a remember instruction. In the present study, all participants were first asked only to recall the second list: the R-words. This way, any F-words that were recalled when asked to recall R-words could be considered intrusions. In line with Rubin et al.'s (2008a) mnemonic model we hypothesized enhanced involuntary recall for trauma-specific material for the Trauma group. More specifically, 
we hypothesized that the Trauma group would mistakenly recall more trauma-specific F-words when asked to recall R-words compared to controls. Furthermore, following Power et al. (2000) we hypothesized that the Trauma group would mistakenly recall more trauma-specific F-words compared to positive, neutral and threatwords, and compared to the controls. Thus the paradigm uses $\mathrm{R}$ and $\mathrm{F}$ instructions, but is not designed to pick up the standard DF effect per se, although it does allow a comparison of correct recall of both F-words and R-words between groups.

The second aim in the present study was to investigate intrusive involuntary recollection of trauma-specific material versus positive, threat-related, and neutral material. In line with Rubin et al.'s (2008a) model, we hypothesized that the trauma group would correctly recall more trauma-specific R-and F-words compared to controls, and compared to positive, neutral and threatrelated words. We also examined the relationship between trauma symptoms and depression symptoms and correct recall of R- and F-words.

\section{MATERIALS AND METHODS PARTICIPANTS}

The Trauma group consisted of 23 women, all of whom had experienced sexual assault in adulthood. The Control group consisted of 23 non-trauma-exposed women. Participant characteristics are presented in Table 1. The participants in the trauma group were recruited from the Emergency Center in Bergen, the Emergency Center in Oslo, Dixi Resource Center, Oslo and from the Center for Crisis Psychology in Bergen. The participants in the control group were recruited with posters placed on public transport, at the University, and in grocery shops. The project was approved by the Regional Committee for Medical Research Ethics for South-Eastern Norway.

\section{DESIGN}

The present study used a mixed design with Group (traumaexposed and non-trauma-exposed controls) as a between-subject factor. The within-subject factors were: Emotional Valence

Table 1 | Mean participant characteristics with associated $\boldsymbol{t}$-values (SD in brackets).

\begin{tabular}{llll}
\hline & Trauma & Control & t(df) \\
\hline Age & $25.26(6.10)$ & $24.08(4.64)$ & $0.73(44)$ \\
Years of post-school & $4.71(2.82)$ & $4.96(1.96)$ & $-0.33(44)$ \\
education & & & \\
Weeks since sexual & $52.61(72.74)$, & - & - \\
assault & range: $4-260$ & & $2.67(44)^{*}$ \\
Number previous & $1.17(14)$ & $0.35(0.49)$ & \\
trauma & & & \\
PDS & $21.43(11.23)$ & $0.73(1.51)$ & $8.76(44)^{* * * *}$ \\
IES & $34.40(18.63)$ & $7.57(13.05)$ & $5.45(41)^{* * * *}$ \\
BDI-II & $18.70(10.48)$ & $5.59(6.93)$ & $5.09(43)^{* * * *}$ \\
BAl & $12.91(8.60)$ & $5.36(6.93)$ & $3.20(42)^{* * *}$ \\
\hline
\end{tabular}

${ }^{*} p<0.05,{ }^{* *} p<0.005,{ }^{* * *} p<0.0001$.
(Trauma-Specific, Threat-related, Neutral, and Positive) and R/F (Remember, Forget). Recall accuracy was the dependent variable.

\section{MATERIALS}

\section{Post-traumatic diagnostic scale}

The post-traumatic diagnostic scale (PDS) is a 49 item self-report scale based on the DSM-IV diagnostic criteria for PTSD. Studies have reported high internal consistency with an alpha coefficient of 0.92 and a test-retest correlation of 0.89 (Foa et al., 1997).

\section{Beck depression inventory-II}

The Beck depression inventory-II (BDI-II) is a self-administered inventory that contains 21 items, it is used to measure current levels of depression (Beck et al., 1996). Beck et al. (1996) reported a test-retest correlation of 0.93 on a sample of 26 clinic patients.

\section{Beck's anxiety inventory}

The Beck's anxiety inventory (BAI; Beck and Steer, 1990) is a 21 item self-report inventory measuring severity of anxiety, which has high internal consistency with an alpha coefficient of 0.92 and a test-retest correlation of 0.75 .

\section{Impact of event scale-revised}

The Impact of event scale-revised (IES-R) is a self-report instrument consisting of 22 items aimed to measure level of stress reactions commonly associated with PTSD: intrusion, avoidance, and hyper-arousal. The internal consistency of the scale is reported to be high; one study reported the intrusion subscale to have an alpha coefficient of 0.91 , the avoidance subscale 0.85 and hyper-arousal 0.90 . Test-retest correlation of 0.57 for intrusion, avoidance 0.51 , and hyper-arousal 0.59 has been reported (Weiss and Marmar, 1997).

\section{Directed forgetting task}

In the present study two lists of words were presented to the participants. Each list consisted of eight positive, eight neutral, eight threat-related, and eight rape-related words. Across participants, the lists served an equal number of times as the Remember-list (R) and the Forget-list (F). To generate these words, seven students were asked to come up with as many as possible words in the four categories. For the threat-related words the students were instructed to come up with words that are associated with traumatic experiences, but not associated with rape (e.g., Traffic accident, Massacre, Fire). Three other students were asked to evaluate the words for emotional content on a scale from -3 to 3 , additionally they were asked to indicate on a scale from 0 to 3 to what degree each word was associated with rape. The frequency of each word was estimated using the Oslo Corpus of Tagged Norwegian Texts database (http://www.tekstlab.uio.no/norsk/bokmaal/english.html). This database consists of 18.3 million words from the most common magazines, newspapers, books, and public reports in Norway. A one-way ANOVA showed that there was no significant difference in frequency between levels of valence, $F(3,76)=0.41$, ns. A oneway ANOVA showed that there was a significant difference in word length between levels of valence, $F(3,76)=0.4 .54, p<0.01$. Words in the neutral valence category had significantly shorter 
word length compared to positive words $t(30)=3.60, p<0.001$, compared to threat-related words $t(30)=2.93, p<0.005$, and compared to trauma-specific words $t(30)=3.46, p<0.001$.

\section{PROCEDURE}

The participants were tested individually in a quiet lab by the first author. The experiment was run by an E-prime script, and consisted of four phases:

1. To-be-forgotten list: The participants were shown an instruction on the screen: "You will now be shown a list of words. Try to remember these words." Following this instruction the participants were presented eight positive words, eight threat-related words and eight trauma-specific words and eight neutral words, in a random order. Each word was presented on a computer screen for $2000 \mathrm{~ms}$, this was followed by an interval of $500 \mathrm{~ms}$ with a blank screen, a fixation cross for $500 \mathrm{~ms}$, and a blank screen for another $500 \mathrm{~ms}$. After the first list of 32 words had been presented, the participants were instructed to try to forget the presented words: "Now try to forget the words you have seen. Try instead to remember the words you will be shown now."

2. To-be-remembered list: The next list of 32 words was then presented on the computer screen, in a random order with the same interval as described above. Eight positive words, eight threat-related words and eight trauma-specific words and eight neutral words were presented.

3. Recall R-words: Participants were then instructed to "Write down as many words as possible from the last list," and were given $2 \mathrm{~min}$ in which to do so.

4. Recall F-words: Participants were asked to: "Write down as many words as possible from the first list." This task also had a time frame of $2 \mathrm{~min}$.

\section{RESULTS}

While the groups did not differ in age or years of education, the Trauma group scored higher on the clinical measures (see Table 1).

\section{CORRECT RECALL OF F- AND R-WORDS}

The mean number of correctly recalled words from the original list was calculated for each word type, and type of instruction (Forget, Remember) as presented in Table 2.

A mixed ANOVA with the between subjects factor of Group (Trauma, Control) $\times$ Instruction (Forget, Remember $) \times$ Valence (Positive, Neutral, Threat-related, Trauma-Specific) was performed. There was a main effect of Instruction $F(1,44)=76.71$, $p<0.001, \eta^{2}=0.60$. There was also a main effect of Valence

Table 2 | Mean number of correctly recalled words (SD in brackets).

\begin{tabular}{llllll}
\hline Word type & \multicolumn{2}{c}{ Trauma } & & \multicolumn{2}{c}{ Control } \\
\cline { 2 - 3 } \cline { 6 - 6 } & Forget & Remember & & Forget & Remember \\
\hline Positive & $0.36(0.58)$ & $1.26(1.18)$ & & $0.87(1.14)$ & $1.78(0.95)$ \\
Neutral & $1.00(1.00)$ & $1.91(1.12)$ & & $1.22(1.41)$ & $2.17(1.19)$ \\
Threat-related & $0.91(0.95)$ & $1.43(1.27)$ & & $0.83(1.92)$ & $2.30(1.18)$ \\
Trauma-specific & $1.13(1.19)$ & $2.34(1.23)$ & & $1.17(0.98)$ & $2.13(1.49)$
\end{tabular}

$F(3,132)=6.62, p<0.001, \eta^{2}=0.14$, due to significantly more neutral words than positive words being recalled $t(45)=3.51$, $p<0.005$, significantly more trauma-specific compared to positive words $t(45)=4.44, p<0.001$, and significantly more threatrelated compared to positive words $t(45)=2.56, p<0.05$. No other comparisons turned out to be significant. No significant effect of Group on recall was found $F(1,44)=2.15, p=0.15$, and none of the interactions involving group were significant, all Fs $<1$.

An additional analysis was carried out after dividing the trauma group into a PTSD group $(n=9)$ and a Trauma group $(n=14)$ according to a recommended cut off score of 27 on the PDS (Griffin et al., 2004). The same pattern of results was observed for this analysis.

\section{F-WORDS MISTAKENLY RECALLED WHEN ASKED TO RECALL R-WORDS}

The mean number of words from the F-list mistakenly recalled when asked to recall R-words was calculated for each word type, these data are shown in Table 3.

A repeated-measures ANOVA with the between subjects factor of Group (Trauma, Control) and the within-subject factor Valence (Positive, Neutral, Threat-related, Trauma-Specific) was performed with the mistakenly recalled F-words. The main effect of Valence was not significant $F(3,132)=0.53$, and there was no main effect of group, $F(1,44)=0.45$. However, there was a significant interaction between Group and Valence, $F(3,132)=4.36$, $p<0.01, \eta^{2}=0.09$. This interaction was broken down by independent samples $t$-tests for each valence, which showed that there was no significant difference between groups for Positive $t(44)=0.00$, Threat-related $t(44)=0.75$, and Neutral words $t(44)=1.04$. However, for Trauma-specific words, the trauma group mistakenly reported more F-words when asked to recall $\mathrm{R}$-words compared to controls, $t(44)=3.14, p<0.005$. Moreover, separate $t$-tests comparing number of mistakenly recalled Forgetwords were computed for each group separately, these analyses showed that the trauma group recalled significantly more Traumaspecific F-words during recall of R-words compared to Neutral F-words $t(22)=3.03, p<0.01$, to Positive F-words $t(22)=2.10$, $p<0.05$, and to Threat-related F-words $t(22)=2.20, p<0.05$. For the control group there were no such significant differences.

Additional analyses were carried out after dividing the trauma group into a PTSD group $(n=9)$ and a Trauma group $(n=14)$. The same pattern of results observed for the trauma group as a whole was also observed for both subgroups.

\section{CORRELATIONS}

Pearson's correlations were performed to investigate the possible relationship between mean numbers of correct recalled F- and

Table 3 | Mean numbers of F-words recalled when instructed to recall R-words only (SD in brackets).

\begin{tabular}{lll}
\hline Word type & Trauma & Control \\
\hline Positive & $0.43(0.66)$ & $0.43(0.66)$ \\
Neutral & $0.32(0.57)$ & $0.52(0.73)$ \\
Threat-related & $0.39(0.58)$ & $0.52(0.59)$ \\
Trauma-specific & $0.87(0.76)$ & $0.26(0.54)$
\end{tabular}


R-words and scores on the IES-R, PDS, BDI, and BAI. Significant correlations between symptom levels and number of correct recalled words were found (see Table 4).

To investigate a possible relationship between the frequency of "intrusive" recall of F-trauma words in the R-list and scores on the IES, PDS, BDI, and BAI, Pearson's correlation was computed. "Intrusive recall" of F-words correlated significantly with symptom levels on the IES, $r=0.31, p<0.05$, the PDS, $r=0.45$, $p<0.005$, and the BDI, $r=0.31, p<0.05$, but not with the BAI, $r=0.26, p=0.09$.

\section{DISCUSSION}

The two aims of the present study were to investigate the modulation of intentional forgetting by valence, and the intrusive recollection of neutral, positive, threat-related, and trauma-specific material in trauma-exposed participants and non-trauma-exposed controls. The results showed that for correct recall significantly more neutral, threat-related, and trauma-specific words were recalled compared to positive words. This pattern was found for both groups, and for both F- and R-words. Higher post-trauma symptom levels, as measured by the IES and the PDS, were associated with fewer correctly recalled R-words. This is in line with previous literature showing that PTSD and depression are associated with lower levels of correct recall of R-words (McNally et al., 1998; Cottencin et al., 2006). Also, significant negative correlations between correct recall of R-words and scores on the BDI and the BAI were found.

In line with our second hypothesis, the trauma-exposed participants mistakenly retrieved more trauma-specific F-words when asked to recall R-words, both relative to controls, and compared to the number of positive, neutral and threat-related F-words they mistakenly recalled. Moreover, a significant correlation between the frequency of "intrusive" recall of F-trauma words and trauma symptoms reported on the IES and the PDS was found. This can be interpreted in line with Power et al.'s (2000) suggestion about a stronger rebound effect for aversive personal-relevant information in depression and PTSD, and moreover the present findings suggest that this also happens in non-clinical groups of trauma-exposed individuals.

The patterns of results in the present study is partly consistent with Ehlers and Clark's (2000) and Brewin's (2001) theories. While these theories predict that voluntary recall of the traumatic

Table 4 | Pearson correlations between correct recall scores and clinical measures.

\begin{tabular}{lllll}
\hline & BDI-II & BAI & PDS & IES \\
\hline R-WORDS & & & & \\
Trauma & -0.39 & -0.41 & $-0.55^{* *}$ & -0.39 \\
Control & -0.37 & -0.27 & -0.24 & -0.18 \\
Total & $-0.42^{* *}$ & $-0.41^{* *}$ & $-0.41^{* *}$ & $-0.32^{*}$ \\
F-WORDS & & & & \\
Trauma & 0.05 & -0.08 & -0.10 & 0.05 \\
Control & $-0.44^{* *}$ & -0.36 & -0.37 & -0.07 \\
Total & -0.18 & -0.24 & -0.15 & -0.04 \\
\hline
\end{tabular}

${ }^{*} p<0.05,{ }^{* *} p<0.01$ event are impaired and involuntary recollection is enhanced, the results in the present study shows a pattern where involuntary recall of trauma-specific material is enhanced, however it was no evidence for impaired voluntary recall of trauma-specific material. The present findings are also partly consistent with Rubin et al.'s (2008a) mnemonic model of PTSD that holds that emotional stress enhances both involuntary and voluntary memory. Thus, similarly to the current findings, this model predicts enhanced involuntary recall of trauma-specific material for the trauma group. However, the model also predicts higher voluntary recall of trauma-specific material, which we did not find in the present study.

The present study gives no support to either a hypothesis about a general or a trauma-specific deficit in intentional forgetting for trauma-exposed individuals, as measured by voluntary recall of Fwords on the DF task. The absence of group differences for correct recall of any word valence for both R- or F-words contrasts with previous findings (Cottencin et al., 2006), suggesting a general deficit in intentional forgetting in PTSD. Our results are more in line with McNally et al.'s (1998) study where the results showed no difference between the groups in recall of trauma-related R-words or F-words. However, in McNally et al.'s (1998) study the PTSD patients demonstrated lower recall rates for positive and negative R-words, whereas this tendency was not found in the present study.

The conflicting results may be caused by differences in design. Former studies (e.g., McNally et al., 1998; Cottencin et al., 2006) have used an item-version of the DF task, whereas the present study employed a modified version of the list-version. In the modified version used in the present study all participants were first asked to recall the R-words, thus output order must be considered more carefully. It is not only the Remember cue that facilitates recall of Rwords, but also the fact that the participants are asked to recall the R-words before the F-words. Thus, order of recall might have been an influencing factor for correct recall of F- and R-words. Moreover, the time between the encoding and recall of R-words was considerably shorter than the time between encoding and recall of F-words. This might have influenced differences in recall rates between F- and R-words. Additionally, in contrast to Cottencin et al.'s (2006) study sample, participants in the present study were not formally assessed for a diagnosis of PTSD. Thus, differences in post-trauma symptoms might have contributed to the conflicting results.

Differences in word length between levels of valence can be argued to have influenced the results in the present study. Words in the neutral valence category had significantly shorter word length compared to positive words, threat-related words, and traumaspecific words However, for correct recall a pattern was found where significantly more neutral, threat-related, and traumaspecific words compared to positive words were recalled. Thus, an explanation based on differences in word length alone does not fit the results in the present study.

How can we explain the increase in involuntary intrusive recollection of trauma-specific words in the trauma group and the simultaneous lack of group differences in intentional forgetting or correct recall of $\mathrm{R}$ - and F-words? The results do not suggest that the trauma-exposed participants have a general problem with intentional forgetting. Furthermore the recall test of the Fwords suggest that they do not have a problem with intentional 
forgetting of trauma-specific material either. However, the trauma group did demonstrate a higher level of intrusive memories of trauma-specific F-words when asked to recall R-words. A sourcemonitoring deficit for trauma-specific material for the trauma group is one possible explanation. Brennen et al. (2007) used the DRM paradigm to study false memories in trauma-exposed participants with and without PTSD. In the DRM task participants are asked to remember lists of words, some of the members of each list are associatively related to a target word that has not been presented, a so-called "critical lure." Participants typically falsely recall or recognize a high number of critical lures. This tendency to produce false memories has been explained in terms of a sourcemonitoring error, more precisely the participants are not able to separate words that they thought about when they saw the words list from words that were actually presented in the list. In Brennen et al.'s (2007) study the results showed that participants with PTSD mistakenly recalled more trauma-specific critical lures in the DRM paradigm compared to trauma-exposed controls. For neutral lists however there was no difference between groups in false recall. Brennen et al.'s (2007) results can be understood as a trauma-specific source-monitoring error in PTSD. The results of the present study can be interpreted in line with this, but suggests that such a deficit also can arise in trauma-exposed individuals without a diagnosis of PTSD.

The present study has some limitations that need to be addressed. The sample in the present study was non-clinical, and perhaps different results would have emerged with a clinical

\section{REFERENCES}

Beck, A. T., and Steer, R. A. (1990). Beck Anxiety Inventory-Manual. San Antonio, TX: Psychological Corporation.

Beck, A. T., Steer, R. A., and Brown, G. K. (1996). Manual for the Beck Depression Inventory, 2nd Edn. San Antonio, TX: Psychological Corporation.

Berntsen, D. (2001). Involuntary memories of emotional events. Do memories of traumas and extremely happy events differ? Appl. Cogn. Psychol. 15, 135-158.

Berntsen, D. (2009). Involuntary Autobiographical Memories. An Introduction to the Unbidden Past. Cambridge: University Press.

Berntsen, D., Rubin, D. C., and Bohni, M. K. (2008). Contrasting models of posttraumatic stress disorder: reply to Monroe and Mineka (2008). Psychol. Rev. 115, 1099-1107.

Brennen, T., Dybdahl, R., and Kapidžić, A. (2007). Trauma-related and neutral false memories in war-induced posttraumatic stress disorder. Conscious. Cogn. 16, 877-885.

Brewin, C. (2001). A cognitive neuroscience account of posttraumatic stress disorder and its treatment. Behav. Res. Ther. 39, 373-393.
Brewin, C. R.,Hunter, E., Carroll, F., and Tata, P. (1996). Intrusive memories in depression: an index of schema activation? Psychol. Med. 26, 12711276.

Cottencin, O., Vaiva, G., Huron, C., Devos, P., Decrocq, F., Jouvent, R., Goudemand, M., and Thomas, P. (2006). Directed forgetting in PTSD: a comparative study versus normal controls. J. Psychiatr. Res. 40, $70-80$.

Ehlers, A., and Clark, D. M. (2000). A cognitive model of posttraumatic stress disorder. Behav. Res. Ther. 38, 319-345.

Foa, E. B., Cashman, L., Jaycox, L., and Perry, K. (1997). The validation of a self-report measure of posttraumatic stress disorder: the Posttraumatic Diagnostic Scale. Psychol. Assess. 9, 445-451.

Griffin, M. G., Uhlmansiek, M. H., Resick, P. A., and Mechanic, M. B. (2004). Comparison of the posttraumatic stress disorder scale versus the clinician-administered posttraumatic stress disorder scale in domestic violence survivors. J. Trauma. Stress 17, 497-503.

Horowitz, M., Wilner, N., and Alvarez, W. (1979). Impact of Event Scale: a measure of subjective stress. Psychosom. Med. 41, 209-218.

sample. Furthermore, no clinical evaluation was made and PTSD symptoms were determined by self-report on the IES and the PDS alone.

It can be argued that different levels of integration of material have influenced the results. The trauma-specific material can be said to be more integrated than the positive, neutral and threatrelated material, and a higher level of integration might influence source-monitoring error. However, this explanation does not fit the pattern observed in the present study, because the control group did not show a tendency for higher level of mistakenly recalled trauma-specific F-words when asked to recall R-words. Another concern in the present study is the low number of correctly recalled words.

In summary the present study found no differences between trauma-exposed participants and controls for correct recall of F-and R-words. However, the trauma group mistakenly recalled more trauma-specific F-words when asked to recall R-words. The results might suggest a source-monitoring deficit for traumaspecific material in trauma-exposed individuals.

\section{ACKNOWLEDGMENTS}

We are grateful to Atle Dyregrov, the staff at Oslo Emergency Center, Bergen Emergency Center, and DIXI for invaluable help with recruiting participants to the study. Thanks to Else-Marie Augusti and Nils Inge Landrø for comments on a previous draft of this manuscript. Our biggest debt is to the participants who gave their time to the project.

Janoff-Bulman, R. (1992). Shattered Assumptions: Towards a New Psychology of Trauma. New York, NY: Free Press.

Johnson, H. (1994). Processes of successful intentional forgetting. Psychol. Bull. 116, 274-292.

McNally, R. J. (2003). Remembering Trauma. Cambridge, MA: Belknap Press/Harvard University Press.

McNally, R. J., Metzger, L. J., Lasko, N. B., Clancy, S. A., and Pitman, R. K. (1998). Directed forgetting of trauma cues in adult survivors of childhood sexual abuse with and without posttraumatic stress disorder. J. Abnorm. Psychol. 107, 596-601.

Moulds, M. L., and Bryant, R. A. (2002). Directed forgetting in acute stress disorder. J. Abnorm. Psychol. 111, 175-179.

Moulds, M. L., and Bryant, R. A. (2005). An investigation of retrieval inhibition in acute stress disorder. $J$. Trauma. Stress 18, 233-236.

Power, M. J., Dalgleish, T., Claudio, V., Tata, P., and Kentish, J. (2000). The directed forgetting task: application to emotionally valent material. $J$. Affect. Disord. 57, 147-157.

Reynolds, M., and Brewin, C. R. (1999). Intrusive memories in depression and post-traumatic stress disorder. Behav. Res. Ther. 37, 201-215.
Rubin, D. C., Berntsen, D., and Bohni, M. K. (2008a). A memory-based model of posttraumatic stress disorder: evaluating basic assumptions underlying the PTSD diagnosis. Psychol. Rev. 115, 985-1011.

Rubin, D. C., Boals, A., and Berntsen, D. (2008b). Memory in posttraumatic stress disorder: properties of voluntary and involuntary, traumatic and non-traumatic autobiographical memories in people with and without PTSD symptoms. J. Exp. Psychol. Gen. 137, 591-614.

Wegner, D. M. (1989). White Bears and Other Unwanted Thoughts: Suppression, Obsession, and the Psychology of Mental Control. London: The Guilford Press.

Weiss, D. S., and Marmar, C. R. (1997). "The impact of event scale-revised," in Assessing Psychological Trauma and PTSD: APractitioner's Handbook, eds J. P. Wilson and T. M. Keane (New York: Guilford Press), 399-411.

Wessel, I., and Merckelbach, H. (2006). Forgetting "murder" is not easier than forgetting "circle": listwise directed forgetting of emotional words. Cogn. Emot. 20, 129-137.

Zoellner, L. A., Sacks, M. B., and Foa, E. B. (2003). Directed forgetting 
following mood induction in chronic posttraumatic stress disorder patients. J. Abnorm. Psychol. 3, 508-514.

Conflict of Interest Statement: The authors declare that the research was conducted in the absence of any commercial or financial relationships that could be construed as a potential conflict of interest.

Received: 28 June 2011; accepted: 29 August 2011; published online: 29 September 2011.
Citation: Blix I and Brennen T (2011) Intentional forgetting of emotional words after trauma: a study with victims of sexual assault. Front. Psychology 2:235. doi: 10.3389/fpsyg.2011.00235

This article was submitted to Frontiers in Cognition, a specialty of Frontiers in Psychology.
Copyright $(5) 2011$ Blix and Brennen. This is an open-access article subject to a nonexclusive license between the authors and Frontiers Media SA, which permits use, distribution and reproduction in other forums, provided the original authors and source are credited and other Frontiers conditions are complied with. 


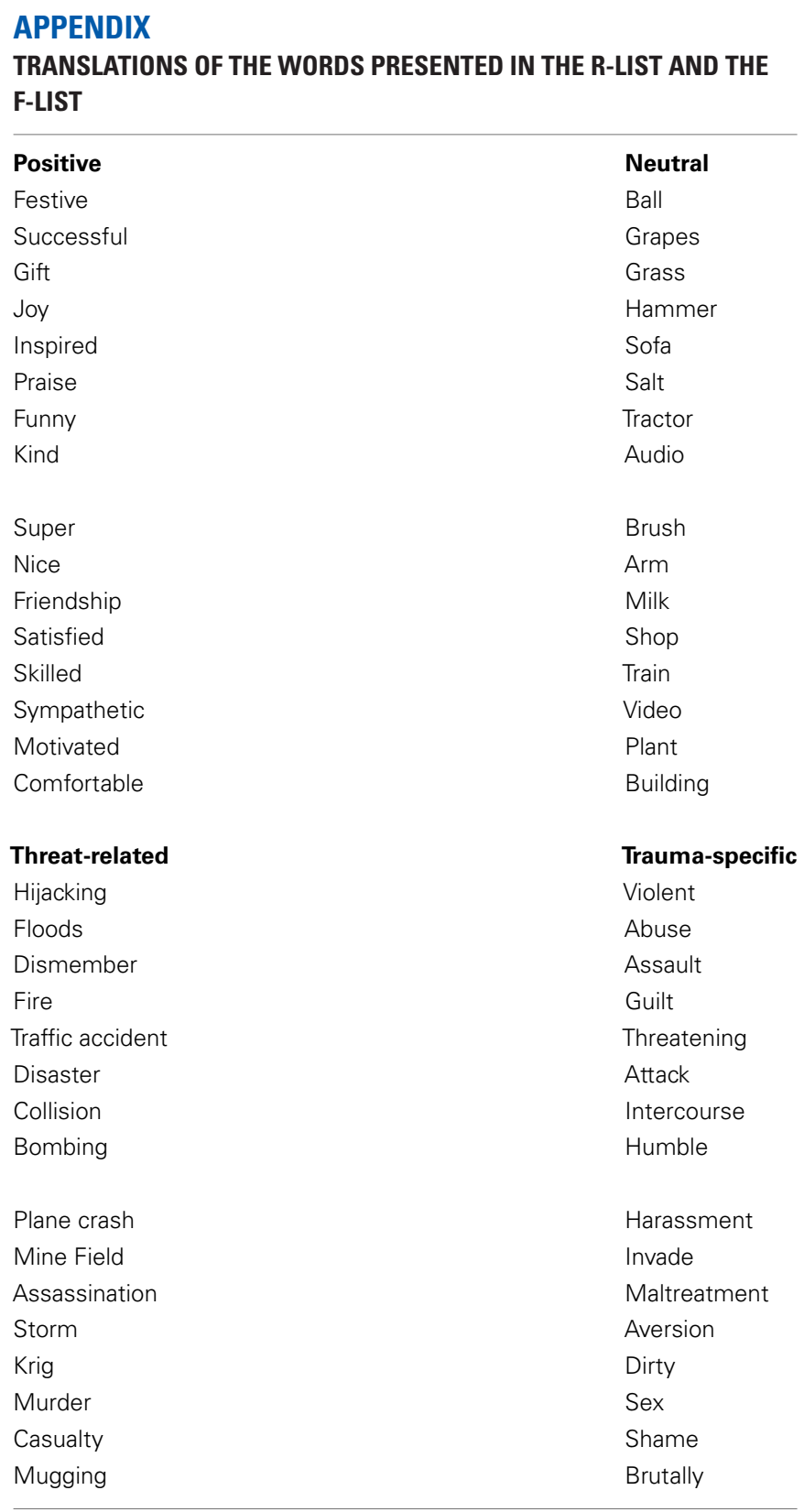

